\title{
Análise de desvios posturais em indivíduos com Doença de Parkinson avaliados pela fotogrametria
}

\author{
Analysis of postural deviations in individuals with \\ Parkinson's disease assessed through photogrammetry
}

\author{
Análisis de desviaciones posturales en personas con \\ Enfermedad de Parkinson evaluadas a través de la \\ fotogrametría
}

\author{
Rita de Cássia Niz Malko ${ }^{1}$, Marina Wolff Branco ${ }^{1}$, Adriano Zanardi da \\ Silva², Bruna Yamaguchi ${ }^{3}$, Vera Lúcia Israel ${ }^{4}$
}

1.Fisioterapeuta. Universidade Federal do Paraná, Curitiba-PR, Brasil.

2.Doutorando no Programa de Pós-Graduação em Educação Física da Universidade Federal do Paraná, Curitiba-PR, Brasil.

3.Doutora pelo programa de Pós-Graduação em Educação Física pela Universidade Federal do Paraná, Curitiba-PR, Brasil

4.Docente do Curso Departamento de Prevenção e Reabilitação em Fisioterapia e Programa de PósGraduação em Educação Física da Universidade Federal do Paraná, Curitiba-PR, Brasil.

\begin{abstract}
Resumo
Introdução. A Doença de Parkinson (DP) consiste numa diminuição nas reservas de dopamina no Sistema Nervoso Central, o que faz com que ocorram alterações físicas e motoras, como alteração da postura corporal e do equilíbrio. A pessoa assume uma postura conhecida como "postura em flexão" que pode levar a déficits em diversas atividades de vida diária. Objetivo. Realizar uma avaliação postural de pacientes com DP por meio da fotogrametria. Método. Foram recrutados indivíduos com DP, classificados entre os estágios 1 a 4 na escala de Hoehn e Yahr. O Software para Avaliação Postural (SAPO) foi utilizado para avaliação postural sendo fotografadas pelas vistas anterior e posterior, com marcadores em determinados acidentes ósseos. A comparação foi entre os hemicorpos e a estratificação pela escala Hoehn e Yahr. Resultados. A avaliação postural de 17 indivíduos (63 111,42 anos) mostrou que que os indivíduos do estágio 3, obtiveram a diferença em todos os pontos analisados, exceto o tendão do calcâneo. Conclusão. Os indivíduos no estágio 3 da DP apresentaram alterações posturais, o que indica um aumento dos desvios posturais em casos mais avançados da doença.
\end{abstract}

Unitermos. Postura; Doença de Parkinson; Fisioterapia; Avaliação de sintomas

\begin{abstract}
Introduction. Parkinson's disease (PD) consists of a decrease in dopamine reserves in the Central Nervous System, which causes physical and motor changes, such as changes in body posture and balance. The person assumes a posture known as "flexed posture" that can lead to deficits in various activities of daily living. Objective. To perform a postural assessment of PD patients using photogrammetry. Method. Individuals with PD, classified between stages 1 to 4 on the Hoehn and Yahr scale, were recruited. The Postural Assessment Software (SAPO) was used for postural assessment, being photographed by the anterior and posterior views, with markers in certain bone accidents. The comparison was between hemibodies and stratification using the Hoehn and Yahr scale. Results. Postural assessment of 17 individuals $(63 \pm 11.42$ years) showed that stage 3 individuals obtained difference in all points analyzed, except for the calcaneus tendon. Conclusion. Stage 3 of PD individuals presented postural changes, which indicates an increase in postural deviations in more advanced cases of the disease.
\end{abstract}

Keywords. Posture; Parkinson's Disease; Physical Therapy; Symptom Assessment 


\section{Resumen}

Introducción. La enfermedad de Parkinson (EP) consiste en una disminución de las reservas de dopamina en el Sistema Nervioso Central, que causa cambios físicos y motores, como cambios en la postura y el equilibrio corporal. La persona asume una postura conocida como "postura flexionada" que puede conducir a déficits en diversas actividades de la vida diaria. Objetivo. Realizar una evaluación postural de pacientes con EP mediante fotogrametría. Metodo. Se reclutaron individuos con EP, clasificados entre las etapas 1 a 4 en la escala de Hoehn y Yahr. El software de evaluación postural (SAPO) se utilizó para la evaluación postural, siendo fotografiado por las vistas anterior y posterior, con marcadores en ciertos accidentes óseos. La comparación fue entre hemibodies y estratificación usando la escala de Hoehn y Yahr. Resultados. Se realizó una evaluación postural de 17 individuos (63 $\pm 11,42$ años) y se encontró que los individuos en etapa 3 obtuvieron una diferencia en todos los puntos analizados, excepto el tendón calcáneo. Conclusión. Las personas en la etapa 3 de la EP mostraron cambios posturales, lo que indica un aumento de las desviaciones posturales en los casos más avanzados de la enfermedad.

Palabras clave. Postura; Enfermedad de Parkinson; Fisioterapia; Evaluación de Síntomas

Trabalho realizado na Universidade Federal do Paraná, Curitiba-PR, Brasil.

\section{INTRODUÇÃO}

A Doença de Parkinson (DP) é uma doença crônica e inflamatória que ocasiona a destruição da mielina no Sistema Nervoso Central (SNC). As manifestações clínicas mais comuns são o comprometimento de equilíbrio, incoordenação, fadiga e fraqueza muscular ${ }^{1}$. Os sintomas podem ser extremamente debilitantes, pois podem progredir com a doença e interferir nas atividades de vida diária. mais frequentes do sistema nervoso central $(\mathrm{SNC})^{1}$. Aproximadamente 1 a $2 \%$ da população acima de 65 anos apresenta DP e tem sido proposto que a doença é uma aceleração anormal do processo de envelhecimento ${ }^{2}$, que consiste numa diminuição nas reservas de dopamina na 
substância negra pars compacta, com presença dos corpúsculos de Lewy, resultando em distúrbios do movimento ${ }^{3}$. De etiologia ainda indefinida, a DP parece estar ligada a fatores genéticos e ambientais ${ }^{4}$ abrangendo um grupo de manifestações motoras e não motoras caracterizadas principalmente pela bradicinesia, tremor em repouso, alteração da postura corporal, do equilíbrio e rigide $z^{5}$. Pode-se encontrar ainda características motoras e não motoras como marcha festinada, diminuição da expressão facial, depressão, alterações cognitivas, alterações da qualidade da voz e distúrbios autonômicos ${ }^{6}$.

Com a evolução da doença, podem ocorrer alterações posturais, assim a pessoa com DP assume uma postura característica, conhecida como "postura em flexão" refletida pela flexão e anteriorização da cabeça, tronco ligeiramente inclinado para frente, semiflexão das articulações de joelhos, quadris e cotovelos ${ }^{7}$.

As alterações de postura podem levar a déficits em diversas atividades de vida diária. Para que se possa iniciar um tratamento com objetivo de promover simetria corporal e consequente melhora da instabilidade postural, é necessário realizar a avaliação postural nestes indivíduos ${ }^{8}$, pois a partir dela podemos observar o alinhamento dos segmentos corporais, criando uma hipótese de distribuição de carga e solicitação mecânica para estruturas como músculos, ligamentos e articulações, tendo uma melhor visão sobre o que acomete este indivíduo. 
A fotogrametria é uma ferramenta de avaliação postural, sendo definida pela American Society of Photogrammetry (ASPRS) ${ }^{10}$ como: a obtenção de informação confiável através de processos de gravação, medição e interpretação de imagens fotográficas que permite a análise biomecânica da postura em posição estática. As vantagens da avaliação postural por meio de softwares incluem o baixo custo e maior replicabilidade na prática profissional, não ser invasivo, resultados de fácil e rápida interpretação clínica ${ }^{8}$.

Assim, sabendo que a avaliação postural faz parte de um grupo de variáveis essenciais a serem avaliados na pessoa com $\mathrm{DP}^{11}$, o objetivo deste estudo foi realizar uma avaliação postural de pacientes com DP por meio da fotogrametria, utilizando o Software para Avaliação Postural (SAPO).

\section{MÉTODO}

\section{Amostra}

Trata-se de um estudo transversal, aprovado pelo Comitê de Ética em Pesquisa do Hospital do Trabalhador/Secretaria Estadual de Saúde do Paraná, sob o número CAAE: 05271512.7.0000.5225.

Foram recrutados indivíduos que atendessem os critérios de inclusão: ambos os sexos, diagnóstico clínico de Doença de Parkinson idiopática, com classificação na escala de Hoehn e Yahr entre os estágios de 1 a 4 , ter 
disponibilidade para participar da pesquisa e concordar com o Termo de Consentimento Livre e Esclarecido (TCLE).

Além disso, foram usados como critério de exclusão indivíduos que não apresentassem marcha independente, relacionada ou não com a $\mathrm{DP}$, ter déficit cognitivo que impossibilite acompanhar instruções verbais ou déficit sensorial visual ou auditivo.

\section{Procedimento}

Os indivíduos foram classificados pela escala de estadiamento da DP proposta por Hoehn \& Yahr ${ }^{12}$, que é amplamente utilizada na prática clínica e em pesquisas ${ }^{13}$. Sua pontuação vai de 1 a 5 , no qual o maior número indica maior comprometimento motor (Quadro 1).

Quadro 1. Estágios da DP segundo a escala de Hoehn \& Yahr ${ }^{12}$.

\begin{tabular}{|l|l|}
\hline Estágio 0 & Nenhum sinal da doença \\
\hline Estágio 1 & Doença unilateral \\
\hline Estágio 2 & Doença bilateral sem déficit de equilíbrio \\
\hline Estágio 3 & Doença bilateral leve a moderada: alguma instabilidade postural \\
\hline Estágio 4 & Incapacidade grave, ainda capaz de caminhar ou permanecer em pé sem ajuda \\
\hline Estágio 5 & Restrito à cama ou cadeira de rodas a não ser que receba ajuda \\
\hline
\end{tabular}

Para a avaliação e mensuração da postura dos pacientes foi utilizado o software para Avaliação Postural (SAPO), um programa de computador gratuito onde seu protocolo é uma proposta de pontos de marcação e medidas para avaliação postural ${ }^{14}$. 
Os pacientes utilizaram roupas apropriadas que permitissem a visualização das marcações (maiô ou sunga), que eram realizadas em acidentes ósseos com marcadores de isopor. O indivíduo foi posicionado na frente de uma parede branca com um fio de prumo ao lado, de modo que os dois ficassem no mesmo plano perpendicular a câmera. Esta ficava localizada a 3 metros de distância do paciente, apoiada num tripé com uma altura de cerca da metade da estatura da pessoa. O indivíduo foi fotografado pelas vistas anterior e posterior, sendo analisados os pontos dos acidentes ósseos: tragus, acrômio, espinha ilíaca ânterosuperior, centro da patela, maléolos laterais e mediais, ângulo inferior da escápula, ponto medial da perna e tendão do calcâneo. A avaliação foi baseada em estudo prévio (Figura 1) e aplicada em indivíduos com DP (Figura 2).

A análise postural foi feita entre os pontos dos dois hemicorpos e foi analisado a diferença de altura de cada acidente ósseo com a preditiva 0 , que significaria que os pontos estão alinhados. Isto é, quanto um lado do corpo pode estar alterado em relação ao 0 , que seria o alinhamento ideal. 


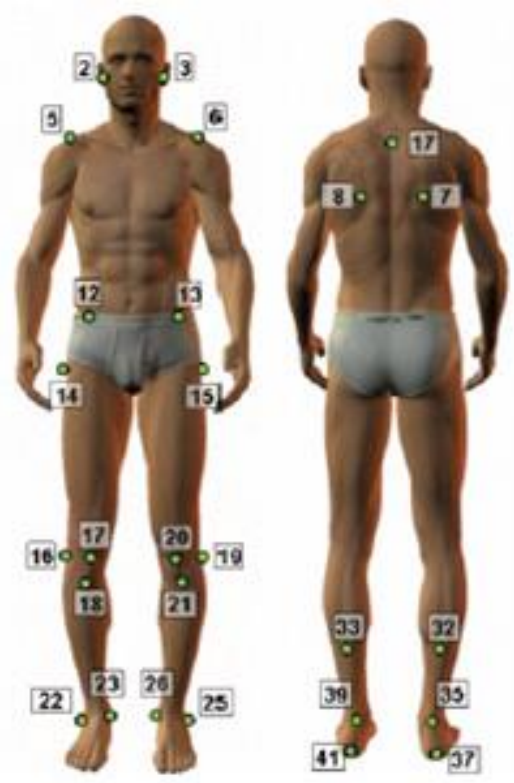

Figura 1. Pontos anatômicos software Sapo (adaptado de SOUZA et al. ${ }^{19}$ )

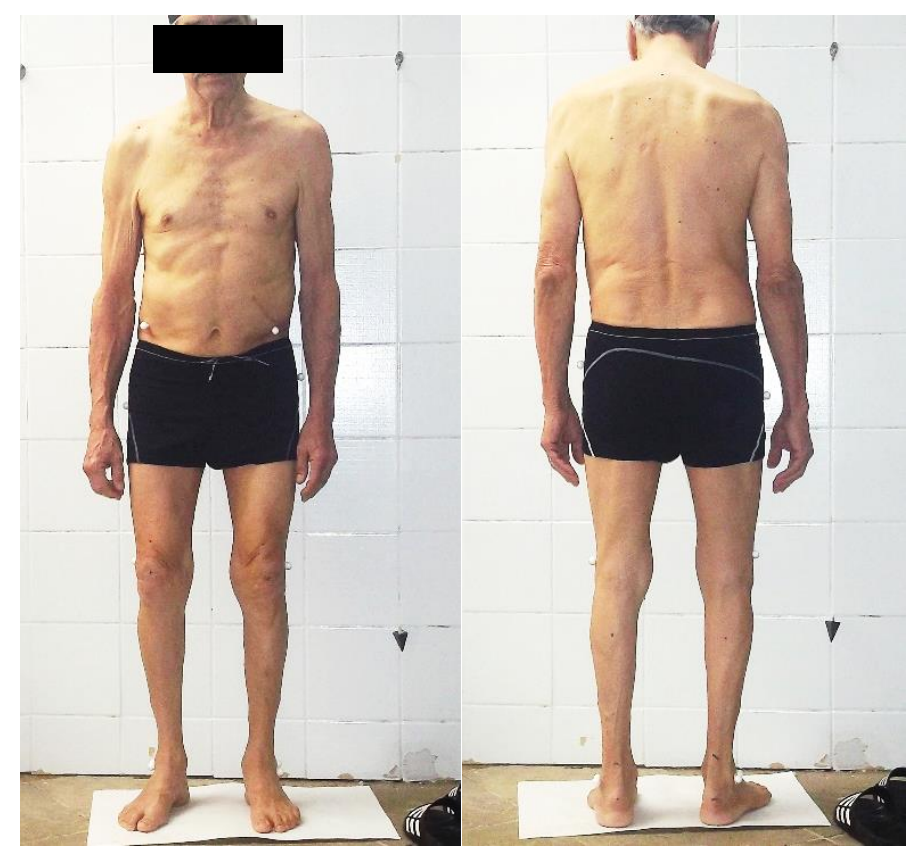

Figura 2. Pontos anatômicos aplicados nos indivíduos com DP (com autorização do indivíduo). 


\section{Análise Estatística}

A análise estatística foi realizada com o auxílio do software SPSS 22. A parametricidade dos dados foram a partir do teste de normalidade Shapiro-Wilk, sendo analisada a diferença de altura dos pontos do hemicorpo direito com 0 esquerdo. A partir disso, as alturas dos pontos deveriam ser comparadas com o preditivo de zero, que indica alinhamento postural, usando então o teste estatístico não paramétrico Mann-Whitney e estratificados pela escala de Hoehn e Yahr.

\section{RESULTADOS}

Foi realizada a avaliação postural de 17 indivíduos, sendo 10 mulheres e 7 homens, que estavam dentro do estágio 2 a 3 da escala de Hoehn e Yahr. A idade média foi de $63 \pm 11,42$ anos. Dos participantes, seis foram classificados no estágio 2 da DP, enquanto três foram classificados no estágio 3 da DP.

Os indivíduos que estavam no estágio 2 não apresentaram diferença no valor da comparativa dos pontos dos dois hemicorpos. Já os indivíduos que estavam no estágio 3, obtiveram a diferença em todos os pontos analisados, exceto o tendão do calcâneo (Tabela 1). 
Tabela 1. Análise dos pontos com a normativa 0 dos indivíduos no estágio 3.

\begin{tabular}{lcc}
\hline \multicolumn{1}{c}{ Acidentes ósseos } & Significância(p) & Diferença da altura dos pontos (cm) \\
\hline Tragus & $\mathbf{0 , 0 1 1}$ & 2,555 \\
Acrômio & $\mathbf{0 , 0 1 7}$ & 2,379 \\
Espinha ilíaca ântero-superior & $\mathbf{0 , 0 1 1}$ & 2,536 \\
Centro da patela & $\mathbf{0 , 0 4 1}$ & 2,041 \\
Maléolos laterais & $\mathbf{0 , 0 4 3}$ & 2,023 \\
Maléolos mediais & $\mathbf{0 , 0 2 6}$ & 2,226 \\
Ângulo inferior da escápula & $\mathbf{0 , 0 2 7}$ & 2,207 \\
Ponto medial da perna & $\mathbf{0 , 0 2 7}$ & 2,214 \\
Tendão do calcâneo & 0,109 & 1,604 \\
\hline
\end{tabular}

\section{DISCUSSÃO}

Estudos sugerem que a idade média de surgimento da doença situa-se entre 58 e 60 anos, embora uma pequena porcentagem possa ser acometida pela doença na faixa dos 40, e até dos 30 anos, assim como demais estudos também apontam tais dados ${ }^{15-17}$. Por isso, a avaliação postural é cada vez mais necessária nessa população, tendo em vista que a instabilidade postural é o fator mais importante associado ao risco de quedas tanto em idosos hígidos como em pessoas com DP ${ }^{18}$.

Os valores angulares do protocolo de avaliação SAPO, na avaliação inter e intraexaminadores são, em sua maioria, confiáveis ${ }^{19}$. Os ângulos propostos para a quantificação das assimetrias posturais utilizados pelo protocolo do software SAPO são satisfatoriamente confiáveis para a grande maioria das medidas angulares estudadas, quando avaliadas por 
examinadores diferentes em um mesmo registro fotográfico ${ }^{19}$.

A DP apresenta alguns sinais e sintomas similares ao processo de envelhecimento. Nesse sentido, estudo indicou que idosos apresentam maior propensão a anteriorização e de lateralização do tronco na postura estática do que indivíduos jovens ${ }^{20}$. Enquanto isso, outro estudo indicou que idosos possuem maiores inclinações laterais e ânteroposteriores, o que contribui para a anteriorização e lateralização do tronco ${ }^{21}$.

As alterações posturais na DP estão associadas, além das disfunções dos sistemas dopaminérgicos, ao processo de envelhecimento, o qual tem associação direta a DP22, devido à perda neural progressiva à medida que a idade avança. $A$ diferença é que no idoso hígido a perda neuronal corresponde a cerca de $4,7 \%$, já na DP essa perda é cerca de $45 \%$, o que pode explicar as alterações posturais encontradas nos indivíduos desse estudo não decorrente apenas das alterações posturais provocadas pela DP, como também por serem indivíduos idosos ${ }^{1}$.

Estudo com o mesmo método de avaliação que o da presente pesquisa, alterações posturais encontradas foram variáveis, porém na DP há uma clara tendência destes indivíduos adotarem uma postura flexora quando estão nos estágios intermediários a avançados da doença ${ }^{23}$.

Estudo similar verificou 10 indivíduos com DP do sexo feminino, com média de idade de 70,5 anos e entre os estágios 1 e 3 da Hoehn e Yahr, foi possível identificar 
alterações posturais tanto em vista lateral (anteriorização cervical, acentuação da cifose torácica e flexão anterior de tronco) como em vista anterior e posterior (desalinhamento das espinhas ilíacas superiores, acrômios e cabeça e inclinação lateral da cervical e do tronco $)^{24}$. Esses achados corroboram com nossa pesquisa, pois também foram verificadas as mesmas alterações posturais dos pontos anatômicos em vista anterior e posterior.

Quando comparamos os achados de alteração postural dos estágios 2 e 3 da Hoehn e Yahr, percebemos que os resultados corroboram com a descrição dos estágios. No estágio 2 da escala a doença é bilateral sem déficit de equilíbrio ${ }^{25}$, e não nos mostra déficit postural significativo desses indivíduos, como nos resultados obtidos no estudo, que não encontrou diferença entre os pontos anatômicos dos indivíduos nesse estágio. Já no estágio 3, a doença é bilateral leve a moderada, com alguma instabilidade postural, o que nos leva a entender que esse indivíduo já possui uma alteração postural que contribui para essa instabilidade ${ }^{25}, 0$ que é presente nesse estudo visto que foi possível identificar alterações posturais pertinentes em todos os pontos anatômicos avaliados (com exceção do tendão do calcâneo) no estágio 3 da doença.

Mesmo com métodos de avaliação postural diferentes, verifica-se que pesquisas indicam que indivíduos com DP apresentam alterações posturais que podem estar associadas com o maior estadiamento da doença $e$ relacionadas com mais elevados risco de quedas ${ }^{23,26}$. Por 
isso, faz-se necessário a avaliação postural para identificar, junto a outros aspectos motores, qual a condição motora e o risco de queda atual dos indivíduos.

Citamos como fatores limitadores do estudo o baixo número de participantes, dificultando a inferência de informações, bem como a ausência de comparação com idosos hígidos, o que poderia revelar diferenças no resultado da avaliação postural para levantar a discussão do quanto a DP interfere na postura além do processo de senescência.

\section{CONCLUSÃO}

Verificou-se que indivíduos no estágio 3 da doença de Parkinson apresentam alterações posturais, o que sugere que desvios e alterações posturais aumentam com 0 progredir da doença.

\section{AGRADECIMENTOS}

O presente trabalho foi realizado com apoio da Coordenação de Aperfeiçoamento de Pessoal de Nível Superior - Brasil (CAPES) - Código de Financiamento 001.

\section{REFERÊNCIAS}

1.Rizzo G, Copetti M, Arcuti S, Martino D, Fontana A, Logroscino G. Accuracy of clinical diagnosis of Parkinson disease. A systematic review and meta-analysis. Neurology 2016;86:566-76.

https://doi.org/10.1212/WNL.0000000000002350

2.Sveinbjornsdottir S. The clinical symptoms of Parkinson's disease. J 
Neurochem 2016;139(Suppl 1):318-24.

https://doi.org/10.1111/jnc.13691

3. Kalia LV, Lang AE. Parkinson's disease. Lancet 2015;386:896-912. https://doi.org/10.1016/S0140-6736(14)61393-3

4.Campos C, Rocha NBF, Lattari E, Paes F, Nardi AE, Machado S. Exercise-induced neuroprotective effects on neurodegenerative diseases: the key role of trophic factors. Expert Rev Neurother 2016;16:723-34. https://doi.org/10.1080/14737175.2016.1179582 5. Mirelman A, Bonato P, Camicioli R, Ellis TD, Giladi N, Hamilton JL, et al. Gait impairments in Parkinson's disease. Lancet Neurol 2019;18:697-708. https://doi.org/10.1016/S1474-4422(19)30044-4

6.Khan AU, Akram M, Daniyal M, Zainab R. Awareness and current knowledge of Parkinson's disease: a neurodegenerative disorder. Int J Neurosci 2019;129:55-93.

https://doi.org/10.1080/00207454.2018.1486837

7.Fukunaga JY, Quitschal RM, Doná F, Ferraz HB, Ganança MM, Caovilla $\mathrm{HH}$. Postural control in Parkinson's disease. Braz J Otorhinolaryngol 2014;80:508-14. https://doi.org/10.1016/j.bjorl.2014.05.032

8. Ferraris C, Nerino R, Chimienti A, Pettiti G, Cau N, Cimolin V, et al. Feasibility of home-based automated assessment of postural instability and lower limb impairments in parkinson's disease. Sensors (Basel) 2019;19:1129. https://doi.org/10.3390/s19051129

9. Mellone S, Mancini M, King LA, Horak FB, Chiari L. The quality of turning in Parkinson's disease: a compensatory strategy to prevent postural instability? J Neuroeng Rehabil 2016;13:39. 10.1186/s12984016-0147-4

10.ASPRS. Guidelines for procurement of professional aerial imagery, photogrammetry, lidar and related remote sensor-based geospatial mapping services. Photogramm Eng Remote Sensing 2009;75:134665.http://www.asprs.org/a/society/committees/standards/Procureme nt Guidelines $w$ accompanying material.pdf

11.Opara JA, Małecki A, Małecka E, Socha T. Motor assessment in parkinson's disease. Ann Agric Environ Med 2017;24:411-5. https://doi.org/10.5604/12321966.1232774

12. Hoehn MM, Yahr MD. Parkinsonism: onset, progression, and mortality. Neurology 1967;17:427-42.

https://doi.org/10.1212/wnl.17.5.427

13.Tiago MSF, Almeida FO, Santos LS, Veronezi RJB. Instrumentos de avaliação de qualidade de vida na doença de Parkinson. Rev Neurocienc 2010;18:538-43. https://doi.org/10.34024/rnc.2010.v18.8437

14.Ferreira EAG, Duarte M, Maldonado EP, Burke TN, Marques AP. Postural assessment software (PAS/SAPO): Validation and reliabiliy. Clinics 2010;65:675-81. http://dx.doi.org/10.1590/S180759322010000700005

15.Wong-Yu IS, Mak MK. Task- and Context-Specific Balance Training Program Enhances Dynamic Balance and Functional Performance in Parkinsonian Nonfallers: A Randomized Controlled Trial with Six-Month Follow-Up. Arch Phys Med Rehabil 2015;96:2103-11. http://dx.doi.org/10.1016/j.apmr.2015.08.409 
16.Strouwen C, Molenaar EALM, Keus SHJ, Munks L, Heremans E, Vandenberghe $\mathrm{W}$, et al. Are factors related to dual-task performance in people with Parkinson's disease dependent on the type of dual task? Parkinsonism Relat Disord 2015;23:23-30.

http://dx.doi.org/10.1016/j.parkreldis.2015.11.020

17.Conradsson D, Löfgren N, Nero H, Hagströmer M, Ståhle A, Lökk J, et al. The Effects of Highly Challenging Balance Training in Elderly With Parkinson's Disease. Neurorehabil Neural Repair 2015;29:827-36. http://dx.doi.org/10.1177/1545968314567150

18. Kimmell K, Pulusu VK, Bharucha KJ, Ross ED. Postural instability in Parkinson Disease: To step or not to step. J Neurol Sci 2015;357:14651. http://dx.doi.org/10.1016/j.jns.2015.07.020

19.Souza JA, Pasinato F, Basso D, Correa ECR, Silva AMT. Biofotogrametria confiabilidade das medidas do protocolo do software para avaliação postural (SAPO). Rev Bras Cineantropometria e Desempenho Hum 2011;13:299-305.

http://dx.doi.org/10.1590/S1980-00372011000400009

20.Fonseca LCS, Scheicher ME. Relação entre projeção do centro de gravidade e equilíbrio em idosos. Ter Man 2012;10:440-3. https://repositorio.unesp.br/bitstream/handle/11449/114861/ISSN16 775937-2012-10-50-440-443.pdf?sequence $=1$

21.Nunes ADM, Fonseca LCS, Scheicher ME. Comparação das inclinações lateral e anteroposterior no equilíbrio estático entre jovens, adultos e idosos. Rev Bras Geriatr e Gerontol 2013;16:813-20.

https://doi.org/10.1590/S1809-98232013000400015

22.Geroin C, Nonnekes J, Vries NM, Strouwen C, Smania N, Tinazzi $\mathrm{M}$, et al. Does dual-task training improve spatiotemporal gait parameters in Parkinson's disease? Park Relat Disord 2018;55:86-91. http://dx.doi.org/10.1016/j.parkreldis.2018.05.018

23.Santos SM, Silva RA, Terra MB, Almeida IA, Melo LB, Ferraz HB. Balance versus resistance training on postural control in patients with Parkinson's disease: a randomized controlled trial. Eur J Phys Rehabil Med 2017;53:173-83. http://dx.doi.org/10.23736/S19739087.16.04313-6

24.Souza A de, Barreto MM, Santos SMS. Avaliação da Atividade Funcional e Qualidade de Vida em Mulheres com Doença de Parkinson. Rev Saúde e Pesqui 2010;3:167-72.

https://periodicos.unicesumar.edu.br/index.php/saudpesq/article/vie $\mathrm{w} / 1470$

25.Strouwen C, Molenaar EALM, Münks L, Keus SHJ, Zijlmans JCM, Vandenberghe $W$, et al. Training dual tasks together or apart in Parkinson's disease: Results from the DUALITY trial. Mov Disord 2017;32:1201-10. http://dx.doi.org/10.1002/mds.27014

26.Saleh MSM, Rehab NI, Aly SMA. Effect of aquatic versus land motor dual task training on balance and gait of patients with chronic stroke: a randomized controlled trial. NeuroRehabilitation 2019;44:485-92. http://dx.doi.org/10.3233/NRE-182636 